\title{
Asymptotic analysis of a control-oriented open channel flow model
}

\author{
Joni Vasara \\ Intelligent Machines and Systems \\ University of Oulu \\ Oulu, Finland \\ joni.vasara@oulu.fi
}

\author{
István Selek \\ Intelligent Machines and Systems \\ University of Oulu \\ Oulu, Finland \\ istvan.selek@oulu.fi
}

\author{
Enso Ikonen \\ Intelligent Machines and Systems \\ University of Oulu \\ Oulu, Finland \\ enso.ikonen@oulu.fi
}

\begin{abstract}
This paper presents the development and mathematical analysis of an open-channel flow model. A tanksin-series model relying on systems engineering perspectives is constructed. Through a series of assumptions, the obtained nonlinear model is approximated by a linear dynamics, and its asymptotic modal behavior is analysed in infinite dimensions using rigorous mathematical machinery. The analysis exploits the repeating tridiagonal structure of the system matrix using Chebyshev polynomials and elaborates the dependence of the modal dynamics on the so-called flow coefficient, which accounts for the description of the flow of the model. The analysis presented in this paper reveals that using constant flow coefficient for flow description in cascade arrangement of liquid storages results in physically meaningless asymptotic modal behavior. The asymptotic modal behavior is well defined if the flow coefficient is an affine function or higher-order polynomial of the number of elements in the cascade structure.
\end{abstract}

Index Terms-Eigen analysis, modelling, open-channel flow.

\section{A. Nomenclature}

The set of reals, nonnegative reals, positive reals, positive integers and complex numbers are denoted by $\mathbb{R}, \mathbb{R}_{>0}, \mathbb{R}_{+}$, $\mathbb{Z}_{+}$and $\mathbb{C}$ respectively. The symbol $U_{N}(x)$ denotes the degree $N$ Chebyshev polynomial of second kind, while := represents equality by definition. For vectors $x \in \mathbb{R}^{n}, x^{T}$ denotes the transposition operator.

\section{INTRODUCTION}

Mathematical modeling of open-channel flow has been and continues to be in the focal point of theoretical and applied research for scientific and engineering communities. Dynamic models have been developed for various demands ranging from flood prediction to hydropower generation, providing means for understanding the governing physical phenomena. As of 2021, the utilization of knowledge captured by firstprinciple models is essential in the analysis of the problem of interest. However, with the advent of reasonably priced computing power, dynamical behavior is often studied by means of numerical simulation, while analytic considerations have received considerably less attention.

The outlined research has been carried out within the project entitled: Optimal River Basin Management with Hydropower (ORBM). The financial support and cooperation provided by PVO Vesivoima Oy is gratefully acknowledged by the authors.
One of the governing engineering paradigms that dominate the practice is the "model and simulate" approach. Supported by a wide variety of commercial modeling software, it is common to build the open channel flow modeling on the one-dimensional Saint-Venant equations, which provide an acceptably good tradeoff between modeling accuracy and computational complexity [2]-[7]. The analysis of the resulting model is then carried out by numerical evaluation of the - by modern terminology - digital twin. Although this approach is widespread and seems vital from the perspective of computational performance offered by recent digital hardware, the related tasks, including analysis, parameter identification, and control design, might be prohibitively excessive, depending on the application.

On the contrary, the essence of engineering is not the construction of models of any kind but the construction of control-oriented mathematical representation: the extraction (identification) of the dominating dynamical behavior of the system of interest from first-principles and/or data. Controloriented models are essential in the understanding of complex physical phenomena. With the sacrifice of some accuracy, control-oriented models are usually remarkably plain and representative with undemanding computational complexity.

The amount of material available on control-oriented modeling of open-channel flow is immerse. However, the majority of the related proposals put the construction (e.g.assumptions, formulation of the governing equations, simplifications, etc.) and the utilization of the resulting model for control in the focal point of the research. The mathematical (analytic) analysis of models got considerably less interest and usually favors frequency-domain descriptions [2], [3].

To fill the gap, this paper contributes to theoretical research including a mathematical analysis of a control-oriented open channel flow model. A model of open-channel flow is derived and simplified through linearization using standard engineering machinery. The model development favours state-space representation and proceeds in time-domain. The main contribution of the paper is the study of the asymptotic behavior of the resulting infinite-dimensional linear state-space model. The dependence of flow coefficient on discretization (number of control volumes) and its effect on the modal assembly of 
the system of interest is elaborated.

The paper is organized as follows: section II details the construction of the open-channel flow model using a standard engineering approach. Assumptions, derivation, simplifications for analysis are considered here. Section III presents the mathematical apparatus used for the study of the derived model in infinite dimensions. First, an analytic formula is derived to describe the eigenvalues of the system matrix, and using this, the convergence of dominant poles of the system is elaborated under the dependence of flow coefficient on the discretization. Finally, section IV provides a summary of the presented work and draws the conclusions.

\section{OPEN-CHANNEL FLOW MODEL: AN ENGINEERING} APPROACH

To construct the mathematical model, here a systems engineering approach is adopted, aiming to represent the open channel flow in a canal by hydrodynamic interaction of liquid storages (tanks). Let the canal be divided into $N \in \mathbb{Z}_{+}$ equally sized finite control volumes referred to as tanks. The control volumes are separated by infinitely thin hoovering walls which prevent matter to cross the boundaries of the control volume. Fluid can enter and leave each finite volume beneath the separation walls as depicted in figure 1 and 2 . The elevation profile of the canal on the $(y, z)$ and $(x, y)$ planes are arbitrary. Let parameter $L \in \mathbb{R}_{+}[\mathrm{m}]$ denote the arc length of the elevation profile which shall be referred to as the length of the canal throughout this article. For simplicity, it is assumed that the canal has a rectangular cross section wetted by the fluid and a constant width $w \in \mathbb{R}_{+}[\mathrm{m}]$. Fluid can enter and leave the canal only at the intake (upper) and discharge (lower) boundaries (see figure 1), represented by inflow $Q^{\text {in }}(t) \in \mathbb{R}_{\geq 0}\left[\mathrm{~m}^{3} / \mathrm{sec}\right]$ and outflow $Q^{\text {out }}(t) \in \mathbb{R}_{\geq 0}$ $\left[\mathrm{m}^{3} / \mathrm{sec}\right]$, no external sources (e.g. precipitation, evaporation, spill loss etc.) are considered.

Regular assumptions are considered to model the fluid flow between coupled tanks: (1) fluid is incompressible, (2) flow develops by elevation difference due to gravity, (3) thermal interactions of fluid storages and (in general) thermodynamic effects are neglected. The governing equations are derived using conservation principles for mass and linear momentum. The conservation of mass can be written as

$$
\frac{L w}{N} \frac{\mathrm{d} h_{i}}{\mathrm{~d} t}= \begin{cases}Q^{\mathrm{in}}(t)-Q_{i}(t), & i=2 \\ Q_{i}(t)-Q_{i+1}(t), & i=2, \ldots, N-1 \\ Q_{i}(t)-Q^{\text {out }}(t), & i=N\end{cases}
$$

where $h_{i}(t) \in \mathbb{R}_{>0}[\mathrm{~m}]$ is the (average) height of the fluid column in tank $i, Q_{i}(t) \in \mathbb{R}\left[\mathrm{m}^{3} / \mathrm{sec}\right]$ denotes the fluid flow between tank $i-1$ and $i$ for all $i \in[2, N] \cap \mathbb{Z}_{+}$ and variable $t \in \mathbb{R}_{\geq 0}$ indexes time. Respectively, the vector $h(t):=\left[h_{1}(t), \ldots, h_{N}(t)\right]^{T}$ gives the zero-order-hold $(\mathrm{ZOH})$ approximation of the fluid surface profile in the canal at time $t$.

The conservation of linear momentum is considered in its steady-state form. Here, the law of momentum conservation

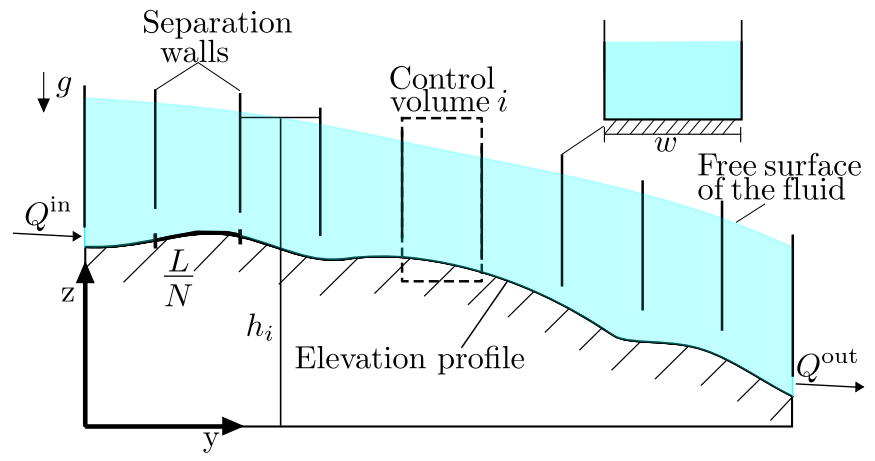

Fig. 1. The arrangement of the tanks-in-series model along the canal.

is represented by the so called valve equation which can be written as follows:

$$
\begin{aligned}
& Q_{i}(t)= \\
& C_{\mathrm{v}, i}(N) \operatorname{sgn}\left(h_{i-1}(t)-h_{i}(t)\right) \sqrt{\left|h_{i-1}(t)-h_{i}(t)\right|}
\end{aligned}
$$

where parameter $C_{\mathrm{v}, i}(N)\left[\mathrm{m}^{2.5} / \mathrm{sec}\right]$ lives under different names within the engineering community including flow coefficient, flow factor or discharge coefficient just to mention a few. The function $\operatorname{sgn}($.$) is the sign function, and |$.$| denotes$ absolute value.

It must be pointed out that in the context of the outlined modeling approach the discharge coefficient $C_{\mathrm{v}}$ has a high impact on the credibility of the obtained model. It captures the fine details of the flow in the canal accounting for the dissipation of energy due to friction. Its analytical form and derivation is far from simple. Its value is usually varying (in time and space) subject to canal geometry, fluid properties, flow conditions and regime (i.e. laminar, turbulent) etc.

Putting (1) and (2) together results in a nonlinear ordinary differential equation (ODE) system of the form

$$
\dot{h}(t)=f\left(h(t), Q^{\text {in }}(t), Q^{\text {out }}(t)\right)
$$

where vector of fluid column heights $h(t):=$ $\left[h_{1}(t), \ldots, h_{N}(t)\right]^{T} \in \mathbb{R}^{N}$ represents the state vector of the system of interest and the boundary conditions

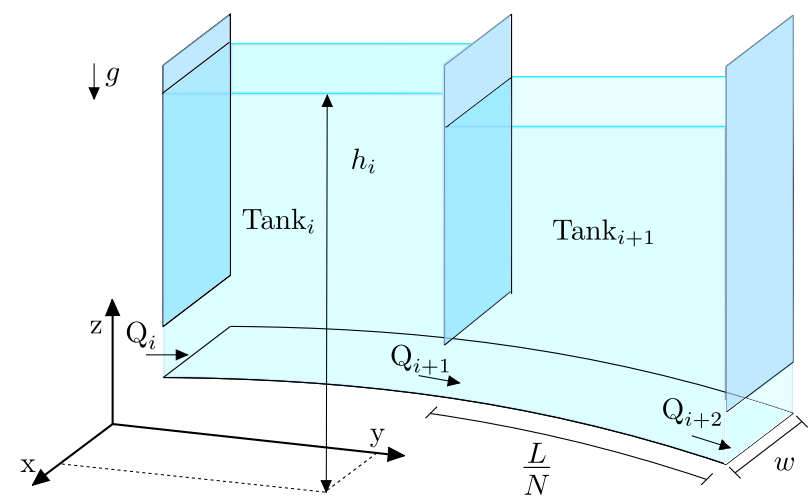

Fig. 2. Control volume representation of a canal section. 
$Q^{\text {in }}(t) \in \mathbb{R}_{\geq 0}, Q^{\text {out }}(t) \in \mathbb{R}_{\geq 0}$ may be regarded as exogenous inputs.

\section{A. Preparation of the model for analysis}

The previous section provided a well-known, standard engineering approach to mathematically capture the dynamical behavior of the system of interest. The resulting model is ready for numerical evaluation once the details get fixed (parameters, geometry, boundary conditions, discharge coefficients, etc.), which undoubtedly involves a tremendous effort.

However, as pointed out already, the author's contribution is the analytical evaluation (analysis) of (3). The analysis aims to understand the asymptotic behavior of the introduced canal model, which involves the study of convergence (divergence) of dynamical behavior as the number of control volumes $N$ goes to infinity. However, once derived, one can observe that the outlined model in its general form is analytically untractable for large $N$; thus, it requires further "treatment" for analytic considerations.

To begin with, let us point out that the square root function which appears on the right-hand side of (2) violates the criteria of Lipschitz continuity at $\left(h_{i-1}(t)-h_{i}(t)\right)=0$ (free surface of the fluid is horizontal in the canal). The lack of the Lipschitz condition introduces an issue regarding the uniqueness of solutions of (3) in case the model is initialized to still water conditions. The problem is resolved by the introduction of the following approximation:

$$
\sqrt{ }(x):=\left\{\begin{array}{cl}
\frac{x}{\sqrt{\delta_{x}}} & \text { for }|x| \leq \delta_{x} \\
\operatorname{sgn}(x) \sqrt{|x|} & \text { otherwise }
\end{array}\right.
$$

where $x \in \mathbb{R}$ and $\delta_{x} \in \mathbb{R}_{+}$is an arbitrarily chosen parameter. The approximation satisfies the Lipschitz criteria by providing a linear approximation of the square root function in the (small) vicinity of zero controlled by the parameter $\delta_{x}$. Additionally, let $z_{F}(y, t)$ denote the free surface (elevation profile) of the fluid. Given these, the following assumptions are introduced:

Assumption 1: The dependence of the discharge coefficient on the location of the control volume can be eliminated by adjusting of the hoovering height of the separation walls.

and

Assumption 2: $\left|z_{F}\left(y_{2}, t\right)-z_{F}\left(y_{1}, t\right)\right| \leq \delta_{x}\left|y_{2}-y_{1}\right|$ for all $y_{1}, y_{2} \in\left[0, y_{L}\right]$ and $t \in \mathbb{R}_{\geq 0}$.

In other words, it is assumed that the flow is subcritical, and the free surface of the fluid is smooth (Lipschitz continuous subject to $\delta_{x}$ ) under the investigated inflow/outflow conditions. This assumption aligns well with the observed behavior of long canals with relatively mild slopes (e.g. natural river, irrigation canal). Consequently, the dynamics of waterfalls, hydraulic jumps, and large amplitude wave propagations are out of the scope of modeling and related analysis.

Putting these together, the flow eqution (2) can be approximated by

$$
Q_{i}(t) \approx \frac{C_{\mathrm{v}}(N)}{\delta_{x}}\left(h_{i-1}(t)-h_{i}(t)\right)
$$

which together with (1) form a linear ODE approximation of the dynamics of the canal:

$$
\dot{h}(t)=\frac{C_{\mathrm{v}}(N) N}{\delta_{x} L w} A h(t)+\frac{N}{L w} B Q(t)
$$

where $A \in \mathbb{R}^{N \times N}, B \in \mathbb{R}^{N \times 2}$ and $Q(t):=$ $\left[Q^{\text {in }}(t), Q^{\text {out }}(t)\right]^{T}$ denotes the vector of exogenous inputs. State transition matrix $A$ is tridiagonal, with the following pattern:

$$
A=\left[\begin{array}{rrrrr}
-1 & 1 & & & \\
1 & -2 & 1 & & \\
& \ddots & \ddots & \ddots & \\
& & 1 & -2 & 1 \\
& & & 1 & -1
\end{array}\right]
$$

where all elements in the main diagonal are -2 except the first and last which are -1 and the off-diagonal elements of the tridiagonal structure are set to one. The control matrix

$$
B=\left[\begin{array}{rr}
1 & 0 \\
0 & 0 \\
\vdots & \vdots \\
0 & 0 \\
0 & -1
\end{array}\right]
$$

In summary, model (6) provides the baseline for the analysis presented in the sequel.

\section{ANALYSIS OF THE MODEL}

The previous section detailed the development and simplification of the open-channel flow model of interest in time domain using state-space representation. This section is devoted to the mathematical analysis of the developed model with respect to its asymptotic behavior, which is obtained by increasing the number of control volumes (thus the dimension of (6)) without bounds.

To achieve this objective, first, the development of the eigenvalues of the system matrix $A$ (appears in (6)) shall be studied with respect to the growth of dimensions. The system matrix preserves the tridiagonal structure (6b) irrespective of its size for all $N \geq 3$, which is utilized during the development of the results. Additionally, the understanding of Chebyshev polynomials is essential to proceed with the calculations in the sequel, thus, let us summarize some of the corresponding results of the related topic:

Remark 1: The Chebyshev polynomial of second kind $U_{N}(x)$ is a univariate polynomial of degree $N$. It satisfies the following equality:

$$
U_{N}(\cos (\theta)) \sin (\theta)=\sin ((N+1) \theta)
$$

which oftentimes used to define $U_{N}(x)$. Additionally, 
Remark 2: The Chebyshev polynomial of second kind $U_{N}(x)$ satisfies the following recurrence relation

$$
U_{N}(x)=2 x U_{N-1}(x)-U_{N-2}(x)
$$

for all $N \geq 2, N \in \mathbb{Z}_{+}$, with initial condition

$$
\begin{aligned}
& U_{0}(x)=1 \\
& U_{1}(x)=2 x .
\end{aligned}
$$

For further details on Chebyshev polynomials see [9].

The importance of Chebyshev polynomials (thus the introduction of the outlined remarks) stems from the fact that these play a fundamental role in the analytic evaluation of determinants of tridiagonal matrices [1]. Similarly to this particular case, tridiagonal matrices frequently appear in physical and engineering applications, embody cascade symmetry, and usually have repeating patterns. These features (if presented in a particular form) can be captured by Chebyshev polynomials which allow the development of analytic formulas quantifying the eigenvalues of the matrix of interest. The analytic representation makes it possible to study the eigen-structure of such matrices in infinite dimensions. Regarding this, the following lemma presents a fundamental result which shall be utilized in the sequel.

Lemma 1: For a tridiagonal matrix $M \in \mathbb{C}^{N \times N}$ of the form

$$
\begin{gathered}
M=\left[\begin{array}{ccccc}
2 x & 1 & & & \\
1 & 2 x & 1 & & \\
& \ddots & \ddots & \ddots & \\
& & 1 & 2 x & 1 \\
& & & 1 & 2 x
\end{array}\right], \quad x \in \mathbb{C} \\
\operatorname{det}(M)=U_{N}(x) .
\end{gathered}
$$

proof. 1: See [1].

Using this, one of the main contributions of this paper is stated by the following lemma:

Lemma 2: The eigenvalues $s_{i}$ of the tridiagonal matrix $A \in \mathbb{R}^{N \times N}$ (defined by (6b)) are

$$
s_{i}=-2-2 \cos \left(\frac{i \pi}{N}\right)
$$

for all $i=1, \ldots, N, N \in \mathbb{Z}_{+}$.

proof. 2: For $N \leq 3$ formula (11) is verified analytically. In these cases, the eigenvalues are: $s_{1}=0(N=1), s_{1}=-2$, $s_{2}=0(N=2)$ and $s_{1}=-3, s_{2}=-1, s_{3}=0(N=3$ respectively) which agree with the statement.

For $N>3$ let us introduce the characteristic polynomial $p_{A}(s)=\operatorname{det}(A-s I)$. Furthermore, let us define a new variable $x$ through the affine mapping $s=-2-2 x$. Then, $p_{A}(x)$ is defined by

$$
p_{A}(x)=\left|\begin{array}{ccccccc}
2 x+1 & 1 & & & & & \\
1 & 2 x & 1 & & & & \\
& 1 & 2 x & 1 & & & \\
& & \ddots & \ddots & \ddots & & \\
& & & 1 & 2 x & 1 & \\
& & & & 1 & 2 x & 1 \\
& & & & & 1 & 2 x+1
\end{array}\right| .
$$

Let $K \in \mathbb{C}^{N \times N}$ denote the matrix appears on the right hand side of (12) and let $K_{1} \in \mathbb{C}^{(N-1) \times(N-1)}, K_{2} \in$ $\mathbb{C}^{(N-2) \times(N-2)}$ be $K$ 's first two principal minors such that

$$
K=\left[\begin{array}{c|c}
2 x+1 & \cdots \\
\hline \vdots & K_{1}
\end{array}\right]=\left[\begin{array}{cc|c}
2 x+1 & 1 & 0 \\
1 & 2 x & \cdots \\
\hline 0 & \vdots & K_{2}
\end{array}\right]
$$

Consequently, $K_{1}\left(K_{2}\right)$ is obtained by removing the first row and column (first two rows and columns) from $K$. Using these, $p_{A}(x)$ can be expressed as

$$
p_{A}(x)=(2 x+1) \operatorname{det}\left(K_{1}\right)-\operatorname{det}\left(K_{2}\right)
$$

by expanding the determinant of $K$ along the first row. The determinants of $K_{1}$ and $K_{2}$ are then computed by expanding along their last row. Utilizing the result provided by lemma 1 the following expressions are obtained:

$$
\begin{aligned}
\operatorname{det}\left(K_{1}\right) & =(2 x+1) U_{N-2}(x)-U_{N-3}(x) \\
& =U_{N-1}(x)+U_{N-2}(x) \\
\operatorname{det}\left(K_{2}\right) & =(2 x+1) U_{N-3}(x)-U_{N-4}(x) \\
& =U_{N-2}(x)+U_{N-3}(x)
\end{aligned}
$$

where (15a) and (15b) are derived by applying the recurrence relation (8) to the expressions defining the determinants (indicated above the numbered equalities determining $\operatorname{det}\left(K_{1}\right)$ and $\operatorname{det}\left(K_{2}\right)$ respectively). By putting (8), (14) and (15) together, the characteristic polynomial $p_{A}(x)$ and the characteristic equation of matrix $A$ in variable $x$ can be expressed as follows:

$$
p_{A}(x) \equiv 2(x+1) U_{N-1}(x)=0 .
$$

The equality above is satisfied in case $U_{N-1}(x)=0$ or $(x+1)=0$. First, let us determine the roots of the Chebyshev polynomial. It follows from (7) that

$$
U_{N-1}(\cos (\theta)) \sin (\theta)=\sin (N \theta) .
$$

Since $\sin (N \theta)=0$ and $\sin (\theta) \neq 0$ for all $\theta=i \pi N^{-1}$ $(i \in 1, \ldots, N-1),(17)$ implies that

$$
U_{N-1}\left(\cos \left(\frac{i \pi}{N}\right)\right)=0
$$

for all $i=1, \ldots, N-1$. Secondly, condition $x+1=0$ yields $x_{N}=-1$. Putting these together, the roots of $p_{A}(x)$ (defined by the characteristic equation (16)) are 


$$
x_{i}=\cos \left(\frac{i \pi}{N}\right), \quad \forall i=1, \ldots, N
$$

due to the fact that $\cos \left(N \pi N^{-1}\right)=-1$ which accommodates the $N$ th $\operatorname{root}\left(x_{N}=-1\right)$. Using (19) and the formerly introduced equality $s_{i}=-2-2 x_{i}$, the roots of the characteristic polynomial $p_{A}(s)$, thus the formula expressing the eigenvalues of $A$ shall result in (11) which completes the proof.

The eigenvalue formula (11) samples the function $s(x)=$ $-2-2 \cos (x)$, on the domain $x \in(0, \pi]$ with sampling instant $\Delta x=\pi N^{-1}$. The graph of $s(x)$ is depicted in figure 3 . Correspondingly, irrespective of the magnitude of the number of control volumes $N$, one root is located at the origin that captures the integrator dynamics of the tanks-in-series model. The rest of the modes exhibit smooth exponential decay as the eigenvalues of $A$ are negative reals between minus four and zero.

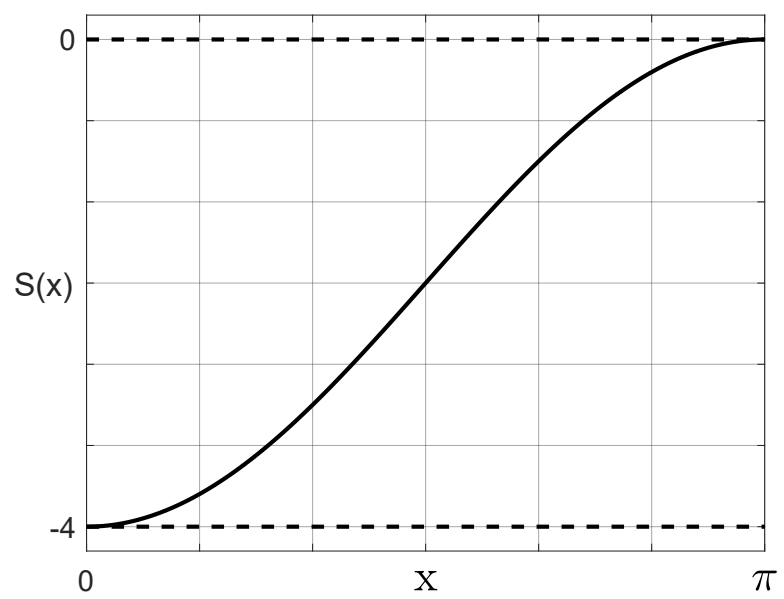

Fig. 3. The graph of the eigenvalue function $s(x)=-2-2 \cos (x)$ of matrix $A$.

\section{A. Asymptotic analysis}

The asymptotic analysis of the model aims to examine the conditions under which (6) has a physically meaningful asymptotic behavior. That is, the convergence of the dynamics of (6) is in the focal point of the analysis as the number of control volumes $N$ goes to infinity. However, convergence is (just) a necessary condition as it is desired that the limit dynamics is interpretable in a physical sense. To achieve vital progress the following assumption is considered:

Assumption 3: The flow coefficient $C_{\mathrm{v}}(N)$ is positive definite and analytic in $N$, for all $N \in \mathbb{Z}_{+}$.

With that said, the modes of (6) are defined by exponential functions $\exp \left(\lambda_{i} t\right)$ where

$$
\lambda_{i}(N)=\frac{C_{\mathrm{v}}(N) N}{\delta_{x} L w}\left(-2-2 \cos \left(\frac{i \pi}{N}\right)\right), \quad i=1, \ldots, N
$$

is obtained by magnifying the eigenvalues of $A$ by a factor determined by geometry and the flow coefficient. Using assumption 3 , it is easy to verify that for any given $i$

$$
\lim _{N \rightarrow \infty} \lambda_{i}(N)=-\infty
$$

Thus, the $i$ th mode of the system of interest represents an infinitely fast dynamics as the number of control volumes increases without bounds. For a canal having physically meaningful dimensions such dynamical behaviour (modes) shall be neglected.

To resolve this issue, let us start counting the modes from "backwards", that is,

$$
\lambda_{N-k}(N)=\frac{C_{\mathrm{v}}(N) N}{\delta_{x} L w}\left(-2-2 \cos \left(\pi-\frac{k \pi}{N}\right)\right)
$$

where $k=0, \ldots,(N-1)$. In systems engineering $\lambda_{N-k}$ are referred to as dominant poles (modes) due to the fact that these represent the largest time constants (slowest convergence rates) in the system's response to inputs. Since $-\lambda_{N-k} \in \mathbb{R}$ the time constant is defined as

$$
T_{N-k}:=3\left|\lambda_{N-k}\right|^{-1}
$$

for all $k=1, \ldots,(N-1)$. Used widely in systems engineering community, by definition, the time constant measures the rate of convergence of a smooth exponential decay. The relation between time constant and convergence rate is inversely proportional: the higher the time constant the slower the convergence and vice versa.

Due to the fact that $-2-2 \cos (x)$ is analytic on $x \in \mathbb{R}$, the eigenvalue function can be stated as

$$
\begin{aligned}
& \left(-2-2 \cos \left(\pi-\frac{k \pi}{N}\right)\right)= \\
& -\left(\frac{k \pi}{N}\right)^{2}+\sum_{i=2}^{\infty}(-1)^{i} a_{2 i}\left(\frac{k \pi}{N}\right)^{2 i}
\end{aligned}
$$

where $a_{2 i} \in \mathbb{R}_{\geq 0}$ for all $i \geq 2, i \in \mathbb{Z}_{+}$. Formula (22) is obtained by deriving the Taylor expansion of $-2-2 \cos (\pi-$ $x$ ) around $x=0$. Using this, the $k$ th (dominant) mode of (6) is determined by

$$
\begin{aligned}
& \lambda_{N-k}(N)= \\
& \frac{C_{\mathrm{v}}(N) N}{\delta_{x} L w}\left(-\left(\frac{k \pi}{N}\right)^{2}+\sum_{i=2}^{\infty}(-1)^{i} a_{2 i}\left(\frac{k \pi}{N}\right)^{2 i}\right)
\end{aligned}
$$

for all $k=0, \ldots(N-1)$. In what follows, for any finite $k \in\{1, \ldots,(N-1)\}$,

$$
\lim _{N \rightarrow \infty} \lambda_{N-k}(N)=\left\{\begin{array}{clll}
0 & \text { if } & \operatorname{deg}\left(C_{\mathrm{v}}(N)\right)=0 \\
\lambda_{N-k}^{\star} & \text { if } & \operatorname{deg}\left(C_{\mathrm{v}}(N)\right)=1 \\
-\infty & \text { if } & \operatorname{deg}\left(C_{\mathrm{v}}(N)\right)>1
\end{array}\right.
$$


where $\operatorname{deg}($.$) denotes polynomial degree and -\lambda_{N-k}^{\star} \in \mathbb{R}_{\geq 0}$ is finite. Note that, here, the counting starts form $k=1$ since the $N$ th dominant pole $\lambda_{N}=0$ irrespective of $N$.

Using (23) and (24) the following conclusions are drawn:

- System (6) has a pole located at zero referred to as integrator mode irrespective of the number of control volumes. The integrator mode represents the massbalance dynamics of a single tank (fluid storage) assuming incompressibility.

- If the flow coefficient $C_{\mathrm{v}}$ is set to a constant value, the dominant modes of (6) approach zero with finer discretization. Consequently, the system's response to changes in boundary conditions shall include modes that exhibit infinitely slow convergence (time constants grow without bounds). The physical behavior corresponding to the dominant modes (excluding the integrator one) is physically meaningless.

- Convergence of the dominant poles (thus convergence of the dynamic behavior) is achieved in case the flow coefficient is an affine function of the number of control volumes. The convergence implies that the asymptotic behavior is physically meaningful.

- Divergence of the dominant poles is observed if the flow coefficient is a second-or-higher order polynomial of the number of control volumes. Consequently, the system's response to changes in boundary conditions shall include one integrator mode while the rest embody infinitely fast convergence (time constants approach zero). From the perspective of dynamical behavior, the infinite-dimensional system shall exhibit simple integrator dynamics and thus can be replaced by a single tank. The asymptotic behavior is physically meaningful.

It must be emphasized that: the derived conclusions target the modal construction of the system of interest. The obtained results are fundamental but preliminary: the study presented here does not consider the weighting and excitation of the modes which play a key role in the formation of the observable dynamics of the system. In order to have a complete picture of the dynamical behavior of (6), the modal controllability and observability aspects shall be included and further studied.

\section{SUMMARY AND CONCLUSIONS}

This paper developed a control-oriented model for openchannel flow and presented a mathematical analysis of its asymptotic behavior. The model development relied on standard systems engineering machinery: open-channel flow in a canal is represented by the dynamics of fluid storages (tanks) in cascade arrangement. The behavior of the fluid is captured by dynamic mass and steady-state momentum conservation laws assuming incompressibility and omitting thermodynamic aspects. The conservation of linear momentum is represented by the widely-known valve equation.

The modeling effort resulted in a nonlinear model having state-space description in time domain. Treated by a series of realistic assumptions, a linear representation of the introduced model is obtained. The state transition matrix of the approximating linear system has a tridiagonal structure, including repeating patterns. This particular feature was captured by Chebyshev polynomials which allowed the development of analytic formula quantifying the eigenvalues of the matrix of interest and, correspondingly, the study of the modal structure of the linear system by increasing the discretization (number of interacting tanks) without bounds referred to as asymptotic analysis.

The asymptotic analysis elaborated the dependence of the modal structure of the system in infinite dimensions on the so-called flow coefficient, which appears in the valve equation determining the flow between tanks. The flow coefficient accounts for the loss of linear momentum due to friction, thus, it is a key ingredient of the developed model. In literature targeting engineering applications it is oftentimes treated as constant. On the contrary, this paper introduces the flow coefficient as a polynomial function of the discretization parameter (number of tanks).

The asymptotic analysis revealed that: (1) used with constant flow coefficient, the tanks-in-series model develops physically meaningless modal behavior as the time constants corresponding to the dominant poles increase without bounds, (2) convergence to a well defined modal behavior is obtained in case the flow coefficient is an affine function of the discretization parameter. The obtained results are fundamental but preliminary: the modal controllability and observability aspects which play a key role in the formation of the observable dynamics of the system shall be further studied.

\section{REFERENCES}

[1] D. Kulkarni, D. Schmidt and S. Tsui, "Eigenvalues of tridiagonal pseudo-Toeplitz matrices,". Linear Algebra and its Applications, Elsevier, 1999, 297, pp.63-80. 10.1016/S0024- 3795(99)00114-7. hal01461924.

[2] Xavier Litrico, V. Fromion. Simplified Modeling of Irrigation Canals for Controller Design. Journal of Irrigation and Drainage Engineering, American Society of Civil Engineers, 2004, 130 (5), pp.373 - 383. (10.1061/(ASCE)0733-9437(2004)130:5(373)). hal-01652556

[3] X. Litrico and V. Fromion, "Infinite dimensional modelling of openchannel hydraulic systems for control purposes," Proceedings of the 41st IEEE Conference on Decision and Control, 2002., Las Vegas, NV, USA, 2002, pp. 1681-1686 vol.2, doi: 10.1109/CDC.2002.1184762.

[4] V. Dalmas, G. Robert, C. Poussot-Vassal, I. Pontes Duff and C. Seren, "From infinite dimensional modelling to parametric reduced-order approximation: Application to open-channel flow for hydroelectricity," European Control Conference (ECC), 2016., Aalborg, Denmark.

[5] J. Schuurmans, 0. H. Bosgra and R. Brouwer, "Open-channel flow model approximation for controller design," Appl. Math. Modelling 1995, Vol. 19, September., in press.

[6] M. Roohi, K. Soleymani, M. Salimi, and M.Heidari,"Numerical evaluation of the general fow hydraulics and estimation of the river plain by solving the Saint-Venant equation“ Modeling Earth Systems and Environment, 2020, 6:645-658

[7] W. Lai and A.A. Khan, "Numerical solution of the Saint-Venant equations by an efficient hybrid finitevolume/finite-difference method", Journal of Hydrodynamics, , 30 (2) (2018), pp. 189-202

[8] V. Dalmas, G. Robert, G.Besancon and D. Georges, "Saint-Venant reduced order model for water level control in open channels", Decision and Control (CDC), 2018., IEEE Conference on Miami Beach, USA pp. 7158-7163, 2018.

[9] J.C. Mason and D.C. Handscom, "Chebyshev Polynomials", CRC Press, 2003. 